\title{
A New Pressure Control Method in the System with Separate Control of Actuator Ports
}

\author{
WANG Di, WANG Junzheng, WANG Shoukun \\ Key Laboratory of Intelligent Control and Decision of Complex System, School of Automation Beijing \\ Institute of Technology, China \\ bit_tom@sina.com
}

\begin{abstract}
Keywords: Separate control of actuator ports; Static operation point; ADRC; Pressure control Abstract. For a valve controlled cylinder speed system with separate control of actuator ports, combining static operation point theory and load sensitivity technology, a new pressure control method is proposed. An optimal pressure based on command speed and load force was the input of pressure controller. At the same time, the output of pressure controller was transformed by inverse proportion, and dynamic pressure control based on error was realized. ADRC (active disturbance rejection control) was used for system simulation. The results show that the new pressure control method is realizable and doesn't need system's mathematic model.
\end{abstract}

\section{Introduction}

From 1987 German professor Backé first proposed the rudiment of separate control technology of actuator ports to now ${ }^{[1]}$, many researchers have made further study of this technology and gotten quite ideal results. In international field, Doctor Erikson Bjorn in Linkoping University mainly studied the application of nonlinear control theory on the system with separate control of actuator ports ${ }^{[2,3]}$. Professor Yao Bin in Purdue University proposed a robust adaptive algorithm to control this type of systems ${ }^{[4]}$. In China, Doctor Liu Yingjie made further study of the system with separate control of actuator ports and proposed feedback linearization algorithm to control the system ${ }^{[5-7]}$. But this algorithm highly depends on the accuracy of system model. So the practical application is restricted by parameter uncertainty and time variation for actual system. Cao Jian in Zhejiang University studied static operation points of the system with separate control of actuator ports and gave the selection principle of static operation points ${ }^{[8]}$. But he did not give a concrete realization method. All in all, researchers mostly use calculated flow feedback algorithm to control the pressure of system, which needs accurate model of the servo valve and a lot of simulation to identify parameters. So the engineering application is restricted. In this paper, a dynamic pressure control method based on error is proposed. It doesn't need system model. And combining with load sensitivity technology, the system can work on an ideal static operation point by changing command pressure.

\section{A dynamic inverse proportion pressure control method based on error}

\section{Theoretical basis}

First we discuss input problem to find out a suitable command pressure. Command pressure is a value when system is on stable state, which has close relationship with the flow conductance of system. The flow conductance of our system is calculated by (1).

$$
K_{\mathrm{eq}}=\frac{w A_{2}}{\sqrt{\mathrm{Nog}_{2} \cdot \frac{F}{A_{2}}}}
$$

Equation (1) bases on the chamber with rod when the cylinder sticks out. $R$ is the ratio of effective area $A_{1}$ of the chamber without rod and effective area $A_{2}$ of another chamber, $F$ is outside force of system, and $p_{z}$ is the pressure of oil source. For our system, $F=B v+\lambda$, where $B$ is viscous damping coefficient and $f$ is load force. On stable condition, the flow conductance only has relationship with speed $v$ and outside force $F$. That is, if these two variables are known, we can get the flow conductance of system. At the same time, the flow conductance of system also has relationship with 
the flow conductance of servo valve. Let $K_{I}$ and $K_{2}$ are the flow conductance of filling valve and delivery valve, we have (2), (3) and (4).

$$
\begin{aligned}
& K_{\mathrm{eq}}=\frac{K_{l} K_{2}}{\sqrt{K_{l}^{2}+R^{3} K_{2}^{2}}}
\end{aligned}
$$

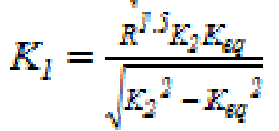

$$
\begin{aligned}
& K_{2}=\frac{K_{P} K_{\mathrm{sq}}}{\sqrt{K_{l}^{2}-R^{3} K_{\varepsilon q}^{2}}}
\end{aligned}
$$

From (2), (3) and (4) we know that for each flow conductance of system, there are infinite pairs of flow conductance $\left(K_{1}, K_{2}\right)$ composed by $K_{1}$ and $K_{2}$. But different pairs of flow conductance have quite large difference on the performance of system. Some pairs of flow conductance even can result in unstable condition. Hence we should find out an optimal flow conductance as the best static operation point, which need consider from the speed sensitivity of system and stability.

For speed sensitivity, we can build an evaluation function $M$ to describe how a pair of flow conductance affects cylinder speed. For example, we let $M=\left|\nabla K_{e q}\right|, \frac{\partial K_{e q}}{\partial K_{I}}=0$ and $\frac{\partial K_{e q}}{\partial K_{2}}=0$, then we can get

$$
K_{I}=R^{0.75} K_{2}
$$

If a pair of flow conductance $\left(K_{I}, K_{2}\right)$ meets the condition of (5), $M$ will be the minimum. It means in this time system speed isn't sensitive to the change of flow conductance. From previous study we know ${ }^{[4]}$ : when the cylinder sticks out, the increase of $\boldsymbol{K}_{2}$ is helpful to reduce speed error. Furthermore, $K_{2}$ won't on sensitive operation point if $K_{l}$ isn't quite large. The pressure difference of a servo valve has relationship with the flow conductance of the servo valve as in (6).

$$
\Delta p=\left(\frac{g}{K}\right)^{2}
$$

We assume that tank pressure is zero. When the cylinder sticks out, pressure of delivery valve $p_{2}$ is equal to $\Delta p$. If command speed $v$ and load force $f$ are known, we can get a flow conductance of system on static condition. Through (5), we can get a pair of flow conductance $\left(K_{1}, K_{2}\right)$ which isn't sensitive to cylinder speed. It means the flow conductance of delivery valve is got. And there is $Q_{2}=A_{2}$, so we can get the pressure of the chamber with rod $p_{2}$. According to above paragraph, if $p_{2}$ decreases, the error of speed control will decrease too.

By a simulation we find if the system has response to step input, the value of back pressure $p_{2}$ has no influence on speed error since speed control is non-error control. But if $p_{2}$ is lower than a value, the system will be unstable. If the system has response to sine input, speed error will decrease as the decrease of $p_{2}$, which is shown in Fig.1. So we need know the upper limit of delivery valve's flow conductance, which is the lower limit of back pressure.

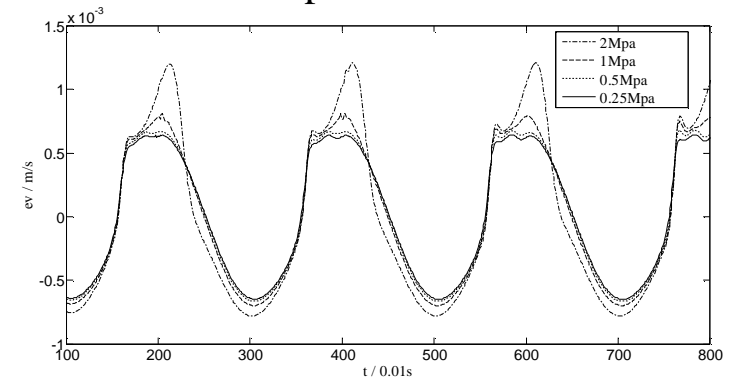

Figure.1 Sine tracking error of cylinder speed (ev) with different back pressure.

According to static operation point theory, when a cylinder sticks out, flow conductance of filling valve and delivery valve should meet following range:

$$
\begin{gathered}
R^{1.5} K_{e q}<K_{1}<K_{1-\max } \\
K_{e q}<K_{2}<K_{2-\max }
\end{gathered}
$$


$K_{I-\max }$ and $K_{2-\max }$, which have relationship with parameters of servo valve, are max flow conductance of the servo valve. And from (6), flow conductance of a servo valve should meet

$$
K=\frac{\varrho}{\sqrt{\Delta p}} \text {. }
$$

When a servo valve is working on rated condition, it has max flow $Q$. And it means the servo valve has the strongest through-flow ability on this moment. Thus the flow conductance $K$ in rated condition is got and looked as max flow conductance of the servo valve. System pressure in max flow conductance can be got by (6). And it is the lower limit of back pressure which makes system stable. In our system, the rated flow of filling valve and delivery valve are $30 \mathrm{~L} / \mathrm{min}$ and $15 \mathrm{~L} / \mathrm{min}, \max \Delta p$ is 21Mpa. By calculation we get that $K_{l-\max }=1.09 \times 10^{-7} \mathrm{~m}^{4} \cdot \mathrm{s}^{-1} \cdot \mathrm{N}^{-0.5}$ and $K_{2-\max }=0.55 \times 10^{-7} \mathrm{~m}^{4} \cdot \mathrm{s}^{-1} \cdot \mathrm{N}^{-0.5}$, which are the upper limit of $K_{I}$ and $K_{2}$. Then we get the lower limit of back pressure with (10).

$$
p_{2-\min }=\left(\frac{w A_{2}}{K_{2-\max }}\right)^{2}
$$

According to practical parameters of our system, the range of rod speed is $0-0.05 \mathrm{~m} / \mathrm{s}$, piston diameter is $25 \mathrm{~mm}$ and rod diameter is $16 \mathrm{~mm}$. By simulation with different rod speed and lower limit of back pressure we got Fig.2.

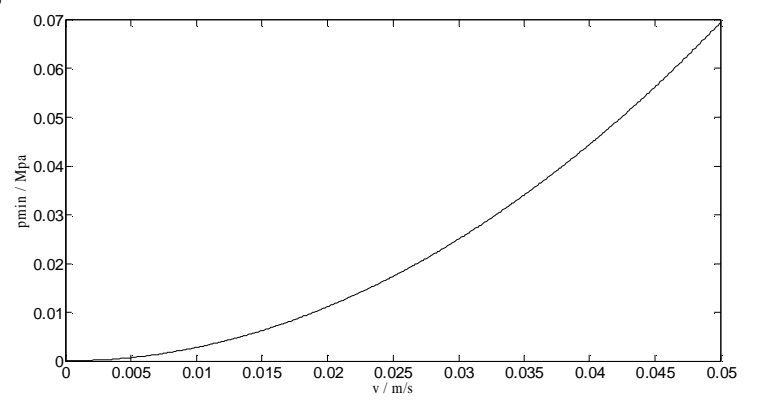

Figure.2 Relationship between rod speed (v) and lower limit of back pressure (pmin)

When rod speed is $0.03 \mathrm{~m} / \mathrm{s}$, back pressure is $0.025 \mathrm{Mpa}$. If a back pressure is lower than that value, the system will be unstable. From Fig. 2 we know, with increase of rod speed, the lower limit will also increase. For practical situation, we let the lower limit be $0.2 \mathrm{Mpa}$. That is, no matter what speed value is, the lower limit of back pressure is all $0.2 \mathrm{Mpa}$.

In summary, outside force $F$ can be got by load sensitivity technology. Then we can get an optimal back pressure value as a command pressure to change static operation point of the system. So the system can be working on an ideal static operation point. It is a combination of load sensitivity technology and static operation point theory.

\section{A new structure of pressure controller based on error}

In previous study, the structure of pressure controller was rarely proposed. Researchers in Zhejiang University proposed a controller structure based on servo valve model [9], which is complicated and needs parameter identification. If the type of servo valve changes, parameters must be calculated again. So it isn't convenient for engineering application.

As we all know, in industrial production field, control method based on error is applied widely for its simple structure and parameters. And there are a lot of control algorithm based on error such as PID and ADRC. It is unrealizable to directly control pressure by error. In simulation we find that the main reason is wrong monotonicity. Control theory based on error gets error $e$ by the difference of output $y$ and input $r$, then it makes $u$ to change output $y$. There is a concealed condition which means $y$ and $u$ must have same monotonicity. According to this, if $e$ is larger, $u$ and $y$ is larger too, the difference of $r$ and $y$ is smaller, so $e$ will decrease. Thus the controller can eliminate error. But if $y$ and $u$ have opposite monotonicity, when $e$ is larger, $y$ is smaller. So $e$ will increase until system is unstable. The relationship of servo valve's flow, pressure and spool displacement are shown in (11).

$$
\mathrm{Q}=C x_{v} \cdot \omega^{*} \sqrt{\frac{2}{p}=\Delta p}
$$


$x_{v}$ is spool displacement, $\omega$ is the gradient of orifice area. $u$ and $x_{v}$ have same monotonicity. Thus if flow is a constant, $u$ and $\Delta p$ are inverse proportion, and if $\Delta p$ is a constant, $u$ and $Q$ are proportional. This is the reason why it is unrealizable to control pressure by error. We added an inverse proportion part to change the monotonicity of $u$ and $y$ with the relationship of $u$ and $e$ unchanged. The structure of pressure controller is shown in Fig.3.

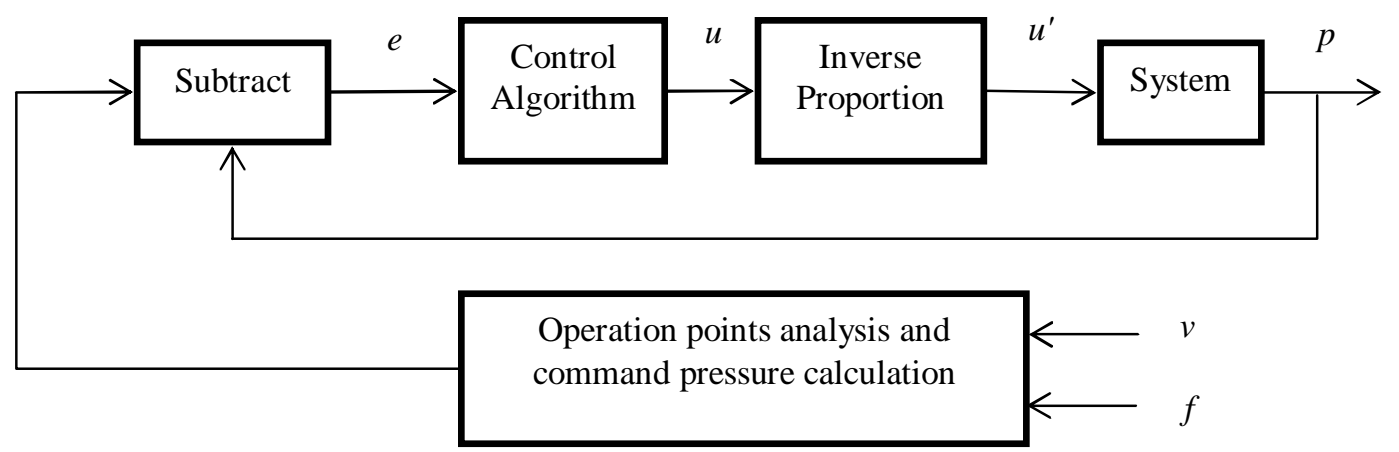

Figure.3 Dynamic inverse proportion pressure controller based on error

All in all, our new pressure control method mainly focuses on controller structure. We proposed a new dynamic pressure control strategy based on error. But control algorithm isn't our innovative point, which is shown in next section.

\section{System composition and algorithm}

\section{System Composition}

First we introduce the composition of valve controlled cylinder speed system with separate control of actuator ports, which is shown in Fig. 4.1 is asymmetric cylinder, 2 is load mass block, 3 and 4 are two independent servo valves, 5 is constant-pressure hydraulic power, 6 is controller, 7 and 8 are pressure sensors, 9 is speed sensor, and 10 is force sensor.

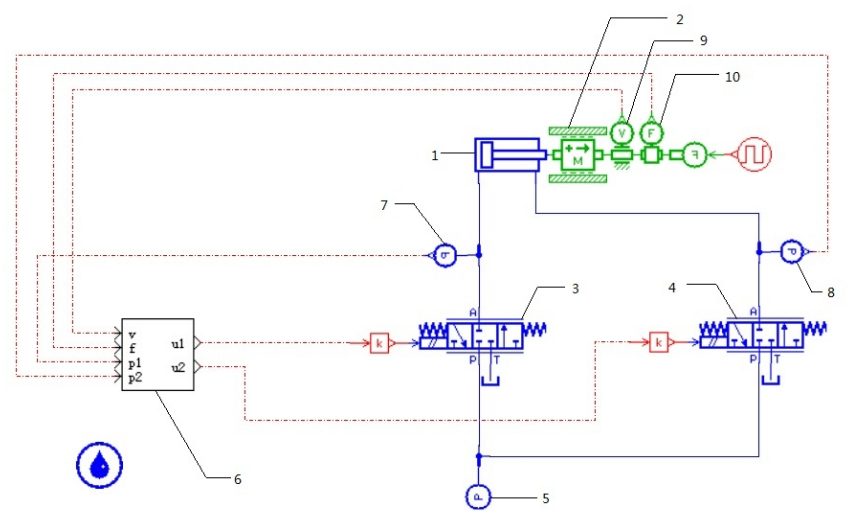

Figure.4 Schematic diagram of valve controlled cylinder speed system with separate control of

\section{ADRC Algorithm} actuator port

The reason why we chose ADRC is as follow: (1) ADRC is an algorithm based on error and it doesn't need high-precision system model; (2) ADRC can inhibit the coupling of two control variables; (3) ADRC is robust so it has the quality of anti-interference.

By analysis of system model, we chose first-order ADRC to control speed and pressure. The structure of first-order ADRC is given as follow ${ }^{[10]}$ :

(1) Tracking Differentiator (TD):

$$
\left\{\begin{array}{c}
v_{1}(h+1)=v_{2}(h)+h \cdot v_{2}(h) \\
v_{2}(h+1)=v_{2}(h)+h \cdot f s t\left(v_{1}(h)-v_{0}, v_{2}(h) r_{2} / h_{0}\right)
\end{array}\right.
$$

$v_{0}$ is reference input. $v_{2}$ is the input whose transient is arranged and $v_{2}$ is its differentiation. $f t\left(x_{1}, x_{2}, x_{2}, h_{0}\right)$ is a function usually used in control and signal processing.

(2) Extended State Observer (ESO): 


$$
\left\{\begin{array}{c}
e=z_{1}(k)-y(h) \\
z_{1}(k+1)=z_{1}(k)+h\left(z_{2}(k)-\beta_{01} e+b u(k)\right) \\
z_{2}(k+l)=z_{2}(h)-h \beta_{02}-f a z(e, \alpha, 0)
\end{array}\right.
$$

$u$ and $y$ are inputs, outputs are estimated values of $\bar{z}_{1}$ and $y . z_{2}$ is the differential estimated value of $y$. The concrete form of function $\mathrm{fal}$ is in (14).

(3) Nonlinear Feedback:

$$
f a l(\epsilon, \alpha, \delta)=\left\{\begin{array}{c}
|e|^{\alpha} \operatorname{sgn}(e),|e|>\delta, \delta>0 \\
e / \delta^{1-\alpha},|e| \leq \delta,
\end{array}\right.
$$

$$
\left\{\begin{array}{l}
a_{1}=v_{1}(h)-z_{1}(h) \\
u_{0}=c \cdot f a l\left(e_{2}, \alpha, \delta\right) \\
u(h)=u_{0}-\frac{z_{2}(h)}{b}
\end{array}\right.
$$

The inputs are TD's output $v_{1}$, ESO's output $z_{1}$ and $z_{2}$. The output is $u$.

\section{Simulation and results}

According to (12), (13) and (15) we designed speed controller and pressure controller. We can debug ADRC parameters by the guidance of [10]. Here we give final controller parameters: (1) Tracking Differentiator, $h=0.01, h_{0}=0.01, r=10$; (2) Speed ADRC Controller, $\beta_{01}=5, \beta_{02}=150, b=5$, $\delta=0.01, c=1, \alpha=0.5$; (3) Pressure ADRC Controller, $\beta_{01}=11, \beta_{02}=60, \delta=11, \delta=0.01, c=1.5, \alpha=0.5$.

We made co-simulation by AMESim and MATLAB, related parameters are shown in Tab.1.

Table.1 System simulation parameters

\begin{tabular}{cccccc}
\hline$A_{2} / \mathrm{m}^{2}$ & $A_{2} / \mathrm{m}^{2}$ & $m / \mathrm{kg}$ & $p_{1} / \mathrm{Mpa}$ & $B / \mathrm{N} \cdot \mathrm{s} \cdot \mathrm{m}^{-1}$ & $\beta_{\mathrm{g}} / \mathrm{Mpa}$ \\
\hline $4.906 \times 10^{-4}$ & $2.897 \times 10^{-4}$ & 20 & 5 & 800 & 870 \\
\hline
\end{tabular}

Simulation time is $10 \mathrm{~s}$. The first is step response, command speed is $0.03 \mathrm{~m} / \mathrm{s}$, load force $f$ is $500 \mathrm{~N}$ from 0 to $5 \mathrm{~s}$ and $100 \mathrm{~N}$ from 5 to $10 \mathrm{~s}$. The second is sine tracking, the amplitude of command speed is $0.01 \mathrm{~m} / \mathrm{s}$, frequency is $0.5 \mathrm{~Hz}$ and load force $f$ is $200 \mathrm{~N}$. Simulation results are shown in following figures.

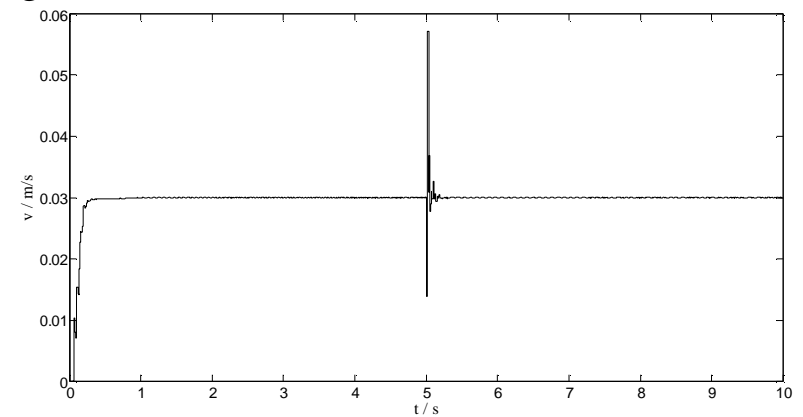

(a) Rod speed (v) vs. time (t)

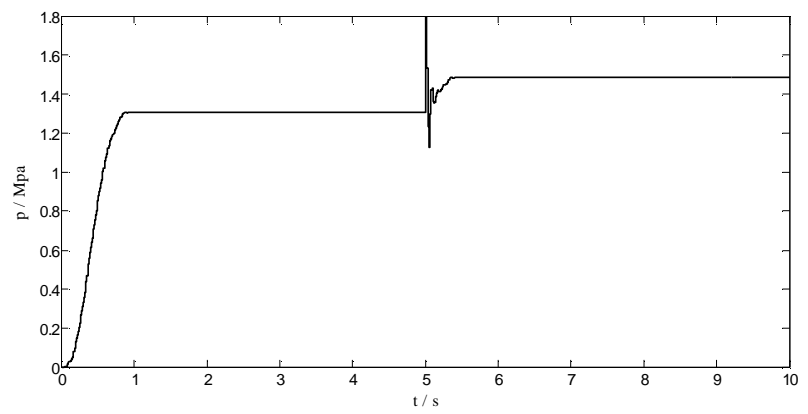

(b) Back pressure (p) vs. time (t)

Figure.5 System response for step input

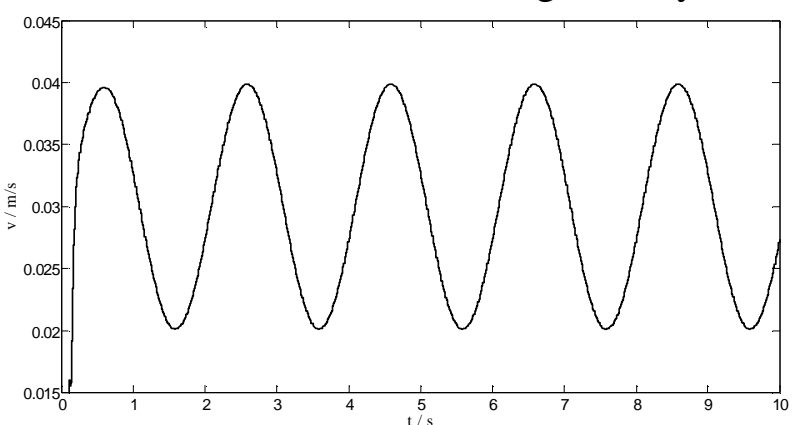

(a) Rod speed (v) vs. time (t)

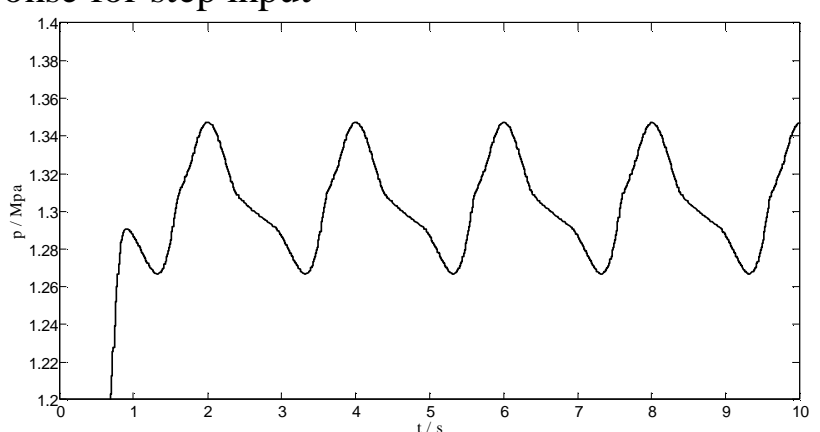

(b) Back pressure (p) vs. time (t)

Figure.6 System response for sine input 
In Fig.5(a), rod speed was kept in $0.03 \mathrm{~m} / \mathrm{s}$ when load force $f$ changed from $200 \mathrm{~N}$ to $-200 \mathrm{~N}$ at $5 \mathrm{~s}$. By calculation we know, if $f$ is $200 \mathrm{~N}$, the optimal back pressure is $1.15 \mathrm{Mpa}$ and when $f$ is $-200 \mathrm{~N}$, the optimal back pressure is $1.35 \mathrm{Mpa}$. In Fig.5(b), back pressure changed to correct value by itself when load force $f$ changed at 5s. Likewise in Fig.6, rod speed could move in desired sine curve and back pressure was corresponding sine curve too. From those two figures we know that our new dynamic pressure control method based on error is correct.

\section{Conclusion}

For the valve controlled cylinder speed system with separate control of actuator ports, a dynamic pressure control principle based on static operation point theory and load sensitivity technology is proposed. In controller structure, an inverse proportion pressure controller base on error is given. It can simplify pressure control processing. The simulation result shows that the new pressure control method is realizable and doesn't need system's mathematic model.

\section{References}

[1] Back e W.. Design systematics and performance of cartridge valve controls, International Conference on Fluid Power. Tampere, Finland, 1987:1-48.

[2] Bjorn E. Control strategy for energy efficient fluid power actuators, Sweden: Linkoping University, 2007.

[3] Bjorn E. Mobile fluid power system design with a focus on energy efficient, Sweden: Linkoping University, 2010.

[4] Yao Bin, Liu Song. Energy-saving control of hydraulic systems with novel programmable valves, Proceedings of the 4th World Congress on Intelligent Control and Automation. Shanghai, China, 2002: 81-91.

[5] Liu Yingjie. Research on key techniques of independent metering directional valve control system, Hangzhou: Zhejiang University, 2011.

[6] Liu Yingjie, Xu Bing, Yang Huayong, et al. Calculation Force Control Based on Linearization Feedback Model of Separate Meter In and Separate Meter Out Control System, Proceedings of The 2009 IEEE International Conference on Automation and Logistics. 2009: 1464-1468.

[7] Liu Yingjie, Xu Bing, Yang Huayong, et al. Modeling of Separate Meter in and Separate Meter out Valve Control System, Proceedings of IEEE/ASME Advanced Intelligent Mechatronics. Singapore, 2009: 227-232.

[8] Cao Jian, Xu Bing, Yang Huayong, et al. Principles for choosing static operation points and simulation analysis of fluid power system based on separate control of actuator ports, J. Chinese Journal of Mechanical Engineering, 2006, 42(6):11-15.

[9] Cao Jian, Xu Bin, Sun Jun, et al. Feedforward compensation controlling based on static operation points in the system with separate control of actuator ports, J. Machine Tool and Hydraulics, 2006, (8): 98-100.

[10] Han Jingqing. From PID technique to active disturbance rejection control technique, J. Control Engineering of China, 2002, 9(3): 13-18. 